\title{
Efectividad del lavado de manos prequirúrgico con cepillo y sin cepillo ${ }^{1}$
}

Institución: Hospital Monseñor Sanabria M.

Fresia Canales Carmona ${ }^{2}$

María de los Ángeles Salazar Campos ${ }^{3}$

\author{
Programa CIEBE-CR
}

\section{COMO CITAR}

\section{RESUMEN}

El objetivo de este artículo es evidenciar la respuesta a una pregunta clínica establecida respecto de la efectividad del lavado de manos prequirúrgico con cepillo y sin cepillo. Esta técnica, disposición obligada y habitual previa a cualquier procedimiento de cirugía, se ha llevado a cabo para disminuir las bacterias transitorias en la piel e inhibir el crecimiento de los microorganismos. Pese a esta medida y de otras como el tratamiento profiláctico con antibióticos, la complicación principal de las cirugías es la infección de la herida quirúrgica, al punto de que en la actualidad es una situación frecuente que origina consecuencias negativas, tanto para las instituciones, como para los pacientes. Dado que elegir el método más efectivo y seguro de lavado de manos prequirúrgico para el binomio personal profesional quirúrgico/ paciente es motivo de controversia, se ejecuta este trabajo, partiendo de la metodología de "práctica clínica de enfermería basada en la evidencia". Se formula una pregunta en formato PICO. Luego, se indaga información en las bases de datos GOOGLE ACADÉMICO, PubMed, Cochrane y Base de Datos para la Investigación en Enfermería (BDIE). Se obtuvo 20 documentos relacionados con el tema de los cuales se descartaron 12 por asuntos metodológicos y de calidad, sólo ocho fueron analizados mediante la aplicación de los criterios que establece CASPe para contestar a la preguntar clínica. Se concluye que el lavado de manos con cepillo es igual de efectivo que la técnica sin cepillo en cuanto a la disminución de la flora bacteriana superficial cutánea, aunque esta última técnica tiene ventajas respecto de la práctica tradicional.

Palabras clave: profesionales-enfermería, lavado-manos-quirúrgico, infecciones-herida-quirúrgica.

\footnotetext{
${ }^{1}$ Fecha de recepción: 8 de agosto del 2013

Fecha de aceptación: 5 de setiembre del 2013

${ }^{2}$ Enfermera. Sala de operaciones del Hospital Monseñor Sanabria. Correo electrónico: nurseop@yahoo.com

${ }^{3}$ Enfermera. Sala de operaciones del Hospital Monseñor Sanabria, Puntarenas. Correo electrónico: marielossalazar@hotmail.es
} 


\title{
Efficiency of the surgical washing of hands with brush and without brush ${ }^{1}$
}

\author{
Fresia Canales Carmona ${ }^{2}$ \\ María de los Ángeles Salazar Campos ${ }^{3}$
}

Institution: Monseñor Sanabria Hospital

CIEBE Program - UCR

\section{CITED}

\begin{abstract}
The purpose of this article is to establish the answer to a clinical question regarding the effectiveness of preoperative hygiene with hand washing brush or without it. This first technique has been performed on the skin to reduce transient bacteria and to inhibit the growth of resident microorganisms as a common and required act before any surgical procedure. Despite this measure and others such as prophylactic antibiotics, the main operative complication continues to be the surgical wound infection. Today, it is as prevalent as in the past with the consequent negative effects derived from it, both for the institutions and for patients. Being controversial today which one is the most effective and safe surgical hand washing method for the binomial: professional surgical team/ patient, this study is done under the criteria of the Evidence-Based Nursing. A question was built in PICO format (Personal, Intervention, Comparison and Outcome). After this is done, continues information search in the databases GOOGLE ACADÉMICO, Pub Med, Cochrane y Base de Datos para la Investigación en Enfermería (BDIE) We obtained 20 articles related to the topic of them 8, which fulfilled the set CASPe criteria to answer the clinical question, were analyzed. We conclude that washing hands with a brush is equally effective as without brush technique in terms of decreased of the superficial skin bacterial flora, although this technique has advantages over the traditional practice.
\end{abstract}

Keywords: professional-nursing, hand-washing-brush, infections-surgical-wound.

\footnotetext{
${ }^{1}$ Date of receipt: Ausgust 8, 2013

Date of acceptance: September 5, 2013

${ }^{2}$ Nurse. Operating room. Monseñor Sanabria Hospital, Puntarenas. E-mail: nurseop@yahoo.com

${ }^{3}$ Nurse. Operating room. Monseñor Sanabria Hospital, Puntarenas. E-mail: marielossalazar@hotmail.es
} 


\section{INTRODUCCIÓN}

La higiene de la piel y especificamente de las manos ha sido objeto de estudio desde hace muchas décadas. Los trabajos de Semmelweis, I. (1846) y de Holmes, O. (1843) son ejemplos del interés por entender la naturaleza de las infecciones y como son transmitidas. Los autores antes mencionados “... relacionaron la naturaleza de la fiebre puerperal y su transmisión a través de las manos de los médicos, la higiene de la piel, y fundamentalmente de las manos, ha sido aceptada como el mecanismo primario de control en la dispersión de agentes infecciosos." (Maimone, S., Castilla, A. 2012, p.1)

De manera permanente, la piel normal es colonizada por bacterias y la mayor concentración se localiza en los estratos más superficiales; dicha flora es transitoria y está asociada a la producción de las infecciones nosocomiales, aunque coexiste con otra flora permanente y profunda que casi no provoca infecciones intrahospitalarias; por tal razón es que "...el lavado de manos con soluciones antisépticas constituye un elemento esencial en el control de las infecciones hospitalarias." (Cuitiño 2011, p. 27).

Existen múltiples evidencias científicas de la necesidad de reducir la flora cutánea superficial, así como la suciedad y residuos de manos y antebrazos para lograr un efecto antimicrobiano durante la cirugía. Este acto para evitar las infecciones postquirúrgicas es tan relevante como la práctica de una técnica idónea en la preparación del área cutánea quirúrgica del paciente, por ello es que Andión (2007) afirma que “...actualmente el lavado de manos, cualquiera sea su tipo, es considerado como la principal medida para reducir la morbimortalidad por infecciones asociadas al cuidado de la salud." (p.1).

Sin enfatizar alguna técnica, no cabe duda de cuán trascendente es el lavado de manos, considerando los protocolos necesarios para su adecuada ejecución; no obstante, cada procedimiento debe adecuarse a la situación para evitar otras consecuencias.

Para reforzar la idea de la relevancia en torno a la higiene prequirúrgica de las manos, se cita a Cuitiño (2011), quien dice que “...las manos de los trabajadores de la salud son el principal mecanismo de transmisión de infecciones entre pacientes. Por lo tanto, la higiene de manos es un componente esencial en cualquier estrategia de control de infecciones." (p.27).

En el hospital Monseñor Sanabria, en Puntarenas, el lavado prequirúrgico se realiza desde siempre - por casi 40 años - con la técnica clásica de cepillado. Esta forma tradicional ha comenzado a cambiar de manera irregular e informal y sin estudio por algún segmento del personal médico quirúrgico, el cual, tras preguntar acerca de la validez y seguridad de la realización del lavado sin cepillado, tan solo respondió " esta es la forma correcta". La anterior motiva a realizar la presente investigación, a lo que se aúna la siguiente opinión:

Si bien el lavado y cepillado prequirúrgico a través del tiempo ha demostrado su eficacia como procedimiento antiséptico, investigaciones recientes mencionan el aumento de las cuentas bacterianas en las manos, secundario a un mayor desprendimiento epitelial resultante del trauma del cepillado, con salida de flora bacteriana, de folículos pilosos y glándulas sebáceas.....dado que los microorganismos proliferan en las manos, dentro del ambiente húmedo de los guantes 
quirúrgicos, problema mayor si los guantes se perforan (Tapia, Reyes, García, Jiménez, Peña, León, 2011, p. 451).

La sepsis postquirúrgica genera complicaciones sociales y administrativas; autores como Plowman (2000) citado por Tanner, Swarbrook, Stuart, (2008) más que conocidas, pero que los enfatizan, confirman que “...las infecciones del sitio quirúrgico provocan retraso de la cicatrización de la herida, aumentan la duración de la estancia hospitalaria, aumentan el uso de antibióticos, provocan dolor innecesario y en casos extremos la muerte del paciente." (p.2).

Para disminuir los factores de riesgo de infecciones en el sitio quirúrgico lo ideal es reducir al máximo la cantidad de bacterias en las manos del cirujano o equipo quirúrgico, previo a la cirugía, y evitar la proliferación de microorganismos por debajo de los guantes. No obstante, pese al cumplimiento de esa acción preventiva, en los años 2010, 2011 y 2012, el Servicio de Bioestadística del hospital Monseñor Sanabria reportó 111 infecciones en heridas quirúrgicas de un total de 9122 cirugías practicadas; 87 de un total de 9942 y 83 de 8331; respectivamente.

En dicho hospital no hay estudios de enfermería basada en la evidencia que permitan la innovación, la seguridad, la economía y la protección del personal ante un cambio de técnica. Las enfermeras (os) del servicio de la sala de operaciones están interesadas en determinar el mejor método basado en la evidencia e implementar su práctica.

El objetivo de este estudio fue establecer si el uso o no del cepillo en el lavado de manos prequirúrgico es igual de efectivo en la reducción de la flora bacteriana como un factor que puede contribuir a la disminución de las infecciones en la herida quirúrgica.

\section{MATERIALES Y MÉTODO}

Para el estudio derivado de esta necesidad detectada por las enfermeras (os) del servicio de la sala de operaciones del hospital Monseñor Sanabria, se formuló una pregunta de primera línea en formato PICO, desglosada de la siguiente manera:

P- Usuario (a): Profesionales de enfermería

I- Intervención que se quiere analizar: Lavado de manos prequirúrgico con cepillo

C- Comparación: Lavado de manos prequirúrgico sin cepillo

O- Resultados clínicos esperados: Igual efectividad en la reducción de la flora bacteriana.

Además, se aplicaron en esta investigación los cinco pasos establecidos de la práctica de enfermería basada en la evidencia:

a) Formular la pregunta de primera línea en el formato PICO.

b) Búsqueda de información científica disponible.

c) Análisis crítico de los datos.

d) Implementación de los resultados encontrados en la práctica profesional. 


\section{Revista Electrónica Enfermería Actual en costa Rica}

e) Evaluación de la implementación.

\section{Pasos establecidos a continuación:}

Se redactó una pregunta clínica de investigación en formato PICO, la cual es planteada de la siguiente manera: El profesional de enfermería que labora en sala de operaciones, que realiza ¿el lavado de manos quirúrgico sin cepillo comparado con el lavado de manos con cepillo, es igual de efectivo para reducir la flora bacteriana?

Como segundo paso se ejecutó una revisión bibliográfica de artículos científicos, con el fin de recuperar la mayor cantidad de documentos acerca de la temática planteada de acuerdo con el tipo de pregunta. Las bases de datos empleadas fueron GOOGLE ACADÉMICO, Pub Med, Cochrane y Base de Datos para la Investigación en Enfermería (BDIE).

Los descriptores empleados para la recuperación de información fueron: "Lavado de manos" OR "Soluciones alcohólicas", "Lavado de manos con cepillo" OR" sin cepillo", "Lavado de manos sin cepillo" AND "Efectividad", "Uso de cepillo en lavado de manos" AND "Cirugía", "Lavado de manos quirúrgico" OR "frotado", "Antisepsia manual quirúrgica" AND "Infección".

En la siguiente etapa, que corresponde al análisis crítico de los datos y la calidad de la información, se aplicó la plantilla de Critical Appraisal Skills Programme Español (CASPe) para responder a la pregunta clínica. Por último, con la mejor información recuperada y analizada se procedió a dar respuesta a la pregunta planteada.

De los 20 artículos identificados, se buscó que cumplieran con los parámetros propios de un artículo científico, dígase, autor, resumen, texto completo y año de publicación. Del total de artículos, 12 fueron excluidos, por razones metodológicas, dado que no cumplían con los requisitos necesarios para la investigación. El resto fue sometido a análisis.

Para la última fase del proceso, basada en la evidencia, considerando los hallazgos en esta revisión, se compartirán y discutirán los resultados con las autoridades y el Comité de infecciones asociadas al cuidado de la salud de la institución para actualizar los procesos relacionados con el lavado prequirúrgico, y finalmente, evaluar tales procesos.

\section{Consideraciones éticas}

En el desarrollo de esta investigación se respetaron los derechos de autor, (aparecen las citas bibliográficas utilizadas), aparte de que el manejo de los datos recolectados se ejecutó con la metodología de práctica de enfermería basada en la evidencia. 


\section{RESULTADOS}

Los documentos analizados fueron los siguientes:

-Irritancy of scrubbing up for surgery with or without a brush (Katsuko Kikuchi-Numagami, Toshio Saishu, Mariko Fukaya, Etsuko Kanazawa and Hachiro Tagami, 1999): Este estudio se refiere a la irritación que causa el uso del cepillo quirúrgico al momento de lavarse las manos. Se trata de un estudio comparativo entre enfermeras, realizado antes y después del lavado de manos diario durante 11 días, en condiciones favorables y desfavorables para la condición de la piel de las manos. Los resultados mostraron que " no se observaron diferencias significativas entre los dos métodos de lavado en el número de colonias bacterianas recogidas de las palmas de las manos antes, inmediatamente después y dos horas después de lavarse las manos"; no obstante, sí aparecieron efectos adversos en la piel, en especial, derivados del uso del cepillo.

-Antisepsia manual quirúrgica con cepillo comparada con antisepsia manual quirúrgica sin utilizar cepillo. (Tanner, Swrbrook, Stuart, 2009). El artículo se desarrolló de acuerdo con el objetivo planteado, el cual pretende comparar el lavado prequirúrgico con y sin cepillo, a partir de ensayos clínicos controlados en dos posibles unidades de asignación al azar para realizar los lavados. Se concluye que ningún ensayo determinó el efecto de utilizar un cepillo durante el lavado quirúrgico; además De que en los lavados acuosos con la clorhexidina al 4\%, dicha sustancia es más efectiva para reducir el número de unidades formadoras de colonias (UFC/ml) en comparación con el povidona iodado. No hubo diferencia entre los lavados alcohólicos utilizados en la preparación del equipo para la cirugía.

-Niveles de satisfacción del equipo quirúrgico entre dos métodos de lavado de manos (Vergara, Morales, Rosales y otros, 2010). En este estudio se compara los niveles de satisfacción, tiempo de lavado, alteraciones dermatológicas y problemas asociados a la colocación de guantes entre el método de lavado de manos tradicional y lavado sin cepillado. Se incluyeron cien cirugías limpias y limpias-contaminadas con cuatrocientos integrantes de los equipos quirúrgicos. Se tomaron cultivos de manos al $20 \%$ de la población, posterior al lavado, y se cuantificó el consumo de agua en miembros del primer grupo. Se evaluó el costo por lavado y la presencia de infecciones de la herida por grupo. Se concluye que el lavado de manos con GCAE - gluconato de clorhexidina al $1 \%$ más alcohol etílico al $61 \%$ - es tan efectivo como el lavado de manos tradicional y se asocia con menor tiempo de lavado, menor resequedad en la piel, menos costo y ahorro de agua.

-Comparación de costo-efectividad del lavado prequirúrgico de manos y antebrazos con diversos antisépticos. (Tapia, Reyes, García, Jiménez, Peña, León, 2011). En este estudio se compara diversos antisépticos de acuerdo con su actividad microbicida, costos y tiempo de procedimiento. Treinta sujetos se lavaron las manos con cloruro de benzalconio, yodopovidona y clorhexidina/ alcohol, durante un lapso de seis días entre cada maniobra. Posterior al lavado prequirúrgico, se obtuvieron muestras para cultivo de la palma de la mano y del lecho unguial, así como después de utilizar guantes estériles por 30 minutos. Se concluye que la clorhexidina/alcohol resultó ser el mejor antiséptico costo-beneficio en relación con la yodopovidona y el benzalconio.

- Eficacia del lavado de manos con solución de base alcohólica versus el lavado de manos estándar con jabón antiséptico (Ruíz, y Urzainqui, 2005). En este ensayo clínico controlado, aleatorio, se compara la reducción en la contaminación de las manos de los profesionales sanitarios tras el uso de jabón antiséptico (JA) y solución de base 
alcohólica (BA). Se constituyeron dos grupos en el estudio, uno para el frotado de manos con una base alcohólica $(\mathrm{N}=13)$ y el otro con jabón antiséptico $(\mathrm{N}=12)$; la asignación de los sujetos fue aleatoria. Durante 2-3 horas de la jornada se monitorizaron cinco actividades que requerían el lavado de manos antes y después de su realización; por ende, se obtuvieron muestras antes y después de cada procedimiento. Los participantes del estudio efectuaron 114 actividades: 59 en el grupo (BA) y 55 en el de (JA). La reducción en el recuento bacteriológico fue más elevada en el grupo del frotado con alcohol (reducción de 83\%), mientras que el grupo de lavado con jabón antiséptico fue del 58\%. Se concluye que el lavado con solución alcohólica es más efectivo que el jabón antiséptico para reducir la contaminación bacteriana de las manos de los trabajadores sanitarios. La literatura revisada se refiere a la lesión cutánea en las manos del personal que cepilla y lava sus manos como acción prequirúrgica de manera continua.

\section{DISCUSIÓN}

Las infecciones en el sitio quirúrgico tiene connotaciones multifactoriales. No obstante, uno de esos factores está relacionado con el cepillado prequirúrgico que se realiza el personal de salud. Por muchas décadas se ha fomentado el uso de cepillos para llevar a cabo este acto con la finalidad de prevenir al máximo el riesgo de infecciones. Sin embargo, las investigadoras han observado que el porcentaje de infecciones en el sitio quirúrgico no ha modificado sus cifras en los últimos años, por lo menos en el Hospital Monseñor Sanabria.

De acuerdo con las referencias consultadas, el lavado con cepillo ha demostrado no estar exento de consecuencias directas tanto para el bienestar del sujeto intervenido quirúrgicamente, como del personal que toma parte en la cirugía. Este lavado provoca un trauma mecánico que libera y dispersa patógenos que pueden producir infecciones en el sitio quirúrgico e incita a la producción de afecciones dermatológicas en el personal de salud. Por otro lado, se ha documentado que las repercusiones derivadas del cepillado se minimizan o desaparecen cuando se prescinde del lavado con cepillo. Tapia et al. (2011) comentan que,

...si bien el lavado y cepillado prequirúrgico a través del tiempo han demostrado su eficacia como procedimiento antiséptico, investigaciones recientes mencionan el aumento de las cuentas bacterianas en las manos secundario a un mayor desprendimiento epitelial resultante del trauma del cepillado, con salida de flora bacteriana de folículos pilosos y glándulas sebáceas. (p. 451).

Además, agregan que "Los microorganismos proliferan en las manos dentro del ambiente húmedo de los guantes quirúrgicos, problema mayor si los guantes se perforan." (p.451). Por otro lado, Corella, Tarragón, Mas y Corella (2007) señalan que no se han encontrado diferencias en relación con el uso de cepillos durante el lavado de manos quirúrgico o prescindiendo de ellos para prevenir infecciones exógenas de las heridas o evidenciar eficacia en eliminar gérmenes. Sin embargo, el uso prolongado o fricción intensa pueden ocasionar microabrasiones en la piel que pueden ser un refugio para los organismos. De igual manera, Villa y Margalejo (2011) señalan que el lavado de manos quirúrgico es una práctica que requiere de fricción y por lo tanto puede dañar la piel y aumentar la dispersión de las bacterias.

Respecto de lo anterior, la dermatitis de contacto irritante es la entidad más frecuente, según Andión $\underline{(2007)}$, 
En ciertos estudios al respecto, aproximadamente el $25 \%$ del personal de enfermería reportó signos y síntomas de dermatitis en sus manos. Se quejan de tener una sensación de sequedad o quemazón, la piel se ve enrojecida y aparece eritema, descamación y fisuras ( p.6).

Las investigadoras han observados que los enfermeros (as) de Sala de Operaciones del Hospital Monseñor Sanabria están experimentando enfermedades cutáneos en las manos, este problema se agudiza con el lavado de manos con los cepillos quirúrgicos que a causa de múltiples re-esterilizaciones sus cerdas se tornan cada vez más duras produciendo escoriaciones en la piel tanto en su estrato superficial como profundo. Este trauma mecánico libera y dispersa patógenos que pueden producir infecciones en el sitio quirúrgico e incita a la producción de afecciones dermatológicas en el personal. Las repercusiones derivadas del cepillado se minimizan o desaparecen cuando se prescinde del lavado con cepillo.

En relación con las afecciones cutáneas del personal que cepilla y lava continuamente sus manos, Maimone y Castilla (2012) afirman que "...frecuentes lavados de manos pueden resultar en daño crónico sobre la piel, dermatitis de contacto irritativa, eczemas y cambio en la flora. La dermatitis irritativa de contacto está asociada con frecuentes lavados de manos." (p. 7).

En cuanto a los potenciales efectos del uso del cepillo en el lavado prequirúrgico y el trauma mecánico que produce en el estrato profundo de la piel, se liga al consenso que hay entre los distintos autores, cuyas publicaciones versan en torno al riesgo de tales acciones, puesto que al liberar bacterias aumenta el riesgo de infección en la herida quirúrgica.

En relación con lo anterior, Tapia y otros (2011) comentan que,

Si bien el lavado y cepillado prequirúrgico a través del tiempo han demostrado su eficacia como procedimiento antiséptico, investigaciones recientes mencionan el aumento de las cuentas bacterianas en las manos secundario a un mayor desprendimiento epitelial resultante del trauma del cepillado, con salida de flora bacteriana de folículos pilosos y glándulas sebáceas (p. 451).

Además, agregan que "Los microorganismos proliferan en las manos dentro del ambiente húmedo de los guantes quirúrgicos, problema mayor si los guantes se perforan." (p.451).

Es de considerar que según Allegranzi, Kilpatrick, Pittet (2011) “...los trabajadores de la salud con dermatitis o lesiones cutáneas en sus manos pueden permanecer colonizados con microorganismos adquiridos por un periodo largo de tiempo.” (p.153). Aunado a esto, Serjan, Saraceni (2005) mencionan que “...cuanto mayor el lavado, el $\mathrm{pH}$ de la piel se incrementa, se reduce el contenido de lípidos, aumenta la pérdida de agua transepidérmica y la colonización bacteriana." (p. 162).

Para contrarestar lo anterior, autores como Peláez, Andrade, y Fereres (2006) citan que, "...el lavado de manos por frotación y cepillado del personal quirúrgico con jabones antisépticos es una de las medidas de eficacia demostrada dirigida a la prevención de la infección intraoperatoria". (p. 186). No obstante, algunos de esos jabones provocan dermatitis, la cual puede ser prevenida si se le da el cuidado que la piel requiere. Para ello 


\section{Revista Electrónica Enfermería Actual en costa Rica}

algunos autores recomiendan que las soluciones “...para frotación de manos con base alcohólica tienen que contener emolientes." (Labadie, Kampf, Lejeunez, et al., 2002, p.3).

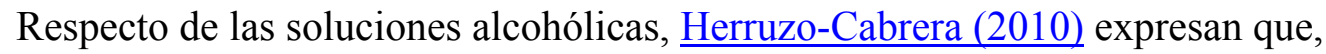

protegen mas la dermis (menor sequedad y menor irritación ) y al aplicarlas, no necesitan cepillado (menor agresión dérmica). La finalidad de su utilización es como con la antisepsia clásica: minimizar el riesgo de infección quirúrgica en caso de rotura de guantes, lo que ocurre hasta en un $18 \%$ de las intervenciones." (p. 266)

Reafirmando la necesidad de una apropiada higiene prequirúrgica, Rincones de Alvarado (2010) señala que es un procedimiento muy efectivo en términos de morbi-mortalidad por infecciones hospitalarias. Por lo cual es fundamental observar como se está realizando en los nosocomios, y tener conciencia de seguir los pasos correctos y el tiempo necesario; respecto de este último aspecto, Viamonte, Viloria, Colmenares, Pin y Zeballos (2009), menciona que "...a pesar que la literatura determina que el lavado de manos pre quirúrgico debe ser entre tres y cinco minutos, no ha sido estandarizado el tiempo para llevar a cabo este procedimiento." (s. p.) Por lo cual tan importante son los aditamentos que se usan como lo es el cepillo o si se prefiere el lavado sin cepillo, como el tiempo de duración del mismo.

Por lo que respecta a la contaminación de la herida quirúrgica es una de las complicaciones más comunes entre las infecciones asociadas a la atención en salud, al respecto Brenner y Nercelles (2011) en su artículo, expresan que,

Pese al creciente conocimiento de las medidas de prevención y control de infecciones (PCI), y aunque la esterilización de instrumental, técnica aséptica, aire limpio y profilaxis antimicrobiana han reducido la incidencia de infecciones de sitio quirúrgico, la tasa se mantiene en niveles inaceptablemente altos. (p. 244)

De igual forma, Álvarez-Gómez (2011), dice que,

Se ha podido constatar que las infecciones nosocomiales generalmente se difunden a través de las manos del personal sanitario y de las demás personas que están en contacto con los pacientes infectados o con superficies situadas en su cercanía, pero el personal médico y paramédico puede convertirse en un posible vehículo de transmisión de éstas. (p. 2)

Otros alegatos que deben considerarse respecto del lavado sin cepillo, aunque no eran parte de los objetivos de esta revisión, son: el ahorro, tanto en tiempo del personal en la práctica del lavado de manos sin cepillo, como en el gasto institucional al obviar el proceso complicado y multipersonal necesario para la reutilización de los cepillos. Sin embargo, las investigadoras consideran que el lavado de manos sin cepillo prequirúrgico debe realizarse de una forma rigurosa para disminuir la flora bacteriana y por ende el riesgo de infección en el sitio quirúrgico. 


\section{Revista Electrónica Enfermería Actual en costa Rica}

\section{CONCLUSIÓN}

A la luz del análisis crítico de la literatura, es claro, -según las premisas de la enfermería basada en la evidencia-, que el lavado de manos quirúrgico con cepillo es tan efectivo como sin cepillo en lo que respecta a disminuir la carga bacteriana superficial cutánea del personal de salud en las salas de operaciones. Sin embargo es importante señalar que la técnica sin cepillo evita el traumatismo repetitivo en las capas profundas de la piel, aspecto que incide en la disminución del riesgo de infección de la herida quirúrgica.

\section{REFERENCIAS}

Allegranzi, B., Kilpatrick, C., Pittet, D. (2011). Higiene de manos. Friedman y Newsom Editores. Conceptos básicos de control de infecciones de IFIC. 2da. edición. Portadown: Editorial International Federation of Infection Control. Recuperado de http://www.theific.org/basic concepts/spanish/IFIC\%20Spanish\%20Book\%202013 PRESS.pdf

Álvarez-Gómez, F. (2011). El lavado de manos. Prevención de infecciones trasmisibles. Gaceta Médica Espirituana, 13(1) Recuperado de http://bvs.sld.cu/revistas/gme/pub/vol.13.(1)_07/p7.html

Andión, E. (2007). Higiene de manos. Lavado de manos y presencia de dermatitis de contacto irritante. Buenos Aires: PRONEO Editorial Panamericana.

Brenner, P., Nercelles, P. (2011). Prevención de infecciones del sitio quirúrgico. Friedman y Newsom Editores. Conceptos básicos de control de infecciones de IFIC. 2da. edición. Portadown: Editorial International Federation of Infection Control. Recuperado de http://www.theific.org/basic_concepts/spanish/IFIC\%20Spanish\%20Book\%202013 PRESS.pdf

Corella-Calatayud, J.M., Tarragón-Sayas, M.A., Mas-Vila, T., Corella-Mas, L. (2007). Infección en cirugía. Aspectos relacionados con enfermería quirúrgica. Enfermería Integral: Revista científica del Colegio Oficial de A.T.S. de Valencia, 80, 7-11. Recuperado de http://www.enfervalencia.org/ei/80/articulos-cientificos/2.pdf

Cuitiño, M. (2011). Nuevas técnicas en el lavado de manos. Medicina preventiva. Buenos Aires: Editorial El caduceo.

Herruzo-Cabrera, R. (2010). Prevención de la infección de localización quirúrgica, según un bundle modificado. Revista española de cirugía ortopédica y traumatología, 54 (5), 265-271. Recuperado de http://www.sciencedirect.com/science/article/pii/S1888441510001049

Kikuchi-Numagami, K., Saishu, T, Fukaya, M., Kanazawa, E y Tagami, H. (1999). Irritancy of Scrubbing Up for Surgery With or Without a Brush. Acta Derm Venereol, 79 (3), 230-232.

Labadie, J.-C., Kampf, G., Lejeunez, B., Exnerx, M., Cottron, O., Girard, R., Orlick, M., Goetz, M.L., Darbord, J.-C., Kramer, A. (2002). Recomendaciones sobre los requisitos para el lavado quirúrgico de manos, puesta en práctica y necesidades para la investigación. Journal of Hospital Infection. European Guidelines, 51, 312-315. doi:10.1053/jhin.2002.1243 Recuperado de http:// www.idealibrary.com 
Maimone, S., Castilla, A. (2012). Lavado de manos y preparación prequirúrgica de la piel. Infección hospitalaria: Infección hospitalaria I. Segunda edición. San Miguel. CODEINEP. Recuperado de http://www.codeinep.org/control/Infeccion_hospitalaria_Icap2.pdf

Peláez, B., Andrade, R., y Fereres, J. (2006). Eficacia microbiológica in vivo de dos cepillos para la asepsia quirúrgica de manos. XII Congreso de la Sociedad Española de Enfermedades Infecciosas y Microbiología Clínica (SEIMC) Comunicaciones 2006, 193.

Rincones de Alvarado, M., (2010). Competencias básicas del cuidado enfermero que reciben los usuarios durante la intervención quirúrgica. Recuperado de http://www.portalesmedicos.com/publicaciones/articles/2382/1/Competencias-basicas-del-cuidado-enfermeroque-reciben-los-usuarios-durante-la-intervencion-quirurgica.html

Ruiz-Delgado, R.Ma., Urzainqui-Zabalsa, O. (2005). Eficacia del lavado de manos con solución de base alcohol estándar con jabón antiséptico. Enfermería Global, 6, 1-5.

Serjan, M.A., Saraceni, L. (2005). Higiene de manos. Rev. Hosp. Mat. Inf. Ramón Sardá, 24 (4), 158-163.

Tanner, J., Swarbrook, S., Stuar, J. (2009). Surgical hand antisepsis to reduce surgical site infection (Review). The Cochrane Library, 1, 1-3. Recuperado de http://onlinelibrary.wiley.com/doi/10.1002/14651858.CD004288.pub2/pdf/abstract

Tapia, J., Reyes, A., García, G., Jiménez C., Peña, J., León, M. (2011). Comparación de costo-efectividad del lavado prequirúrgico de manos y antebrazos con diversos antisépticos. Cir Cir, 79 (5), 447-452. Recuperado de http://www.mediagraphic.com/pdfs/circir/cc-2011/cc115i.pdf

Vergara-Fernández, O., Morales-Olivera, J.M., Ponce-de-León-Rosales, S., Vega-Batista, R., Mejía-Ovalle, R., HuertasJiménez, M. (2010). Niveles de satisfacción del equipo quirúrgico entre dos métodos de lavado de manos. Revista de Investigación Clínica, 62(6), 532-537. Recuperado de http://www.medigraphic.com/pdfs/revinvcli/nn2010/nn106f.pdf

Viamonte, G., Viloria, C., Colmenares, P., Pin, D., Zeballos, R. (2009). Higiene de manos. Tres minutos en lavado de manos. Universidad Gran Mariscal de Ayacucho. 2009.

Villa, S., Margalejo-Raffin, S. (2011). Higiene de manos. Guía de recomendaciones para los establecimientos de salud. ECI, 3 (3), 390-408. Recuperado de http://www.adeci.org.ar/LinkClick.aspx?fileticket=Sfj7egRiMfA\%3D\&tabid=75 